\title{
One-dimensional bigyrotropic magnetic photonic crystals
}

\author{
I. L. Lyubchanskii, ${ }^{\text {a) }}$ N. N. Dadoenkova, M. I. Lyubchanskii, and E. A. Shapovalov \\ Donetsk Physical and Technical Institute of the National Academy of Sciences of Ukraine, \\ 83114, Donetsk, Ukraine
}

A. Lakhtakia ${ }^{\text {b) }}$

Pennsylvania State University, University Park, Pennsylvania 16802-6812

Th. Rasing ${ }^{\text {() }}$

IMM, Radboud University of Nijmegen, 6525 ED Nijmegen, The Netherlands

(Received 21 April 2004; accepted 22 September 2004)

\begin{abstract}
Electromagnetic wave propagation in a one-dimensional magnetic photonic crystal (MPC) made of bigyrotropic magnetic yttrium-iron garnet and nonmagnetic gadolinium-gallium garnet is theoretically investigated using the $4 \times 4$ transfer matrix method. Band gaps in the electromagnetic spectrum are numerically obtained and appear to depend on the helicity and direction of light propagation through the MPC. (C) 2004 American Institute of Physics. [DOI: 10.1063/1.1825060]
\end{abstract}

During the last 15 years, photonic band gap (PBG) materials, or photonic crystals (PCs), have been the objects of intense theoretical and experimental investigation because of promising applications in optoelectronics and telecommunication. ${ }^{1-3}$ These PCs are artificially fabricated structures with one-, two-, or three-dimensional periodic order, with structural periods that are comparable to the wavelength of the incident electromagnetic wave (EMW). ${ }^{1-3}$ The main attraction of PCs is the existence of forbidden regimes or band gaps in their transmittance spectra.

Quite recently, magnetic materials have been incorporated in PCs, which has led to the recognition of new peculiarities of EMW propagation in PCs, e.g., nonreciprocity and unidirectionality, ${ }^{4,5}$ that can be tuned by the application of a magnetic field. In magnetic photonic crystals (MPCs), ${ }^{5}$ forbidden regimes can exist in both the optical and the microwave portions of the electromagnetic spectrum. The PBGs can be found by solving dispersion equations that take into account the periodicity of the permittivity tensor $\epsilon_{j \ell}$ and the permeability tensor $\mu_{j \ell}$.

Optical and magneto-optical effects (MO) in MPCs constructed from yttrium-iron garnet $\mathrm{YIG}\left(\mathrm{Y}_{3} \mathrm{Fe}_{5} \mathrm{O}_{12}\right)$ have been theoretically investigated by several groups, ${ }^{6-8}$ taking into account the magnetization-induced off-diagonal components of the permittivity tensor. Impurity-doped YIG crystals and films, which are transparent in the near-infrared (NIR) regime, are widely used in modern magneto-optics. ${ }^{9}$ For example, recently a miniature tunable broadband magnetooptical modulator based on a Bi-substituted YIG film was presented as the basis of new high-speed magneto-photonic devices. ${ }^{10}$ Also, enhanced Faraday rotation in an all-garnet MPC based on $\mathrm{Bi}_{3} \mathrm{Fe}_{5} \mathrm{O}_{12} / \mathrm{Y}_{3} \mathrm{Fe}_{5} \mathrm{O}_{12}$ multilayers was reported. ${ }^{11}$ A remarkable property of YIG is its bigyrotropy, which is characterized by the fact that in the transparency regime its permittivity tensor and its permeability tensor have comparable contributions to the MO response. ${ }^{12}$ Ageev et $a{ }^{13}$ measured the Faraday rotation at $\lambda$ $=1150 \mathrm{~nm}$ in impurity-doped YIG films with composition $\mathrm{Y}_{3} \mathrm{Fe}_{3.88} \mathrm{Sc}_{0.68} \mathrm{Ga}_{0.44} \mathrm{O}_{12}$, and showed that the off-diagonal

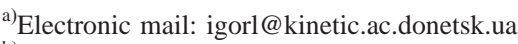

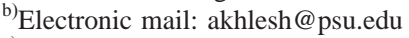

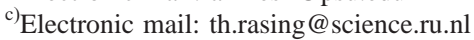

components of both tensors have comparable effects. So, the correct description of wave propagation in YIG-containing structures requires that the solution of the Maxwell equations incorporate both the permittivity and the permeability tensors. ${ }^{14,15}$ In this letter, we investigate PBG effects in YIGMPCs taking the bigyrotropy of YIG into complete account and find that the presence of band gaps strongly depends on the helicity and direction of the EMWs, leading to tunable, polarization selective magneto-photonic elements.

Let us consider a one-dimensional periodic structure composed of bigyrotropic (BG) YIG layers of thickness $d_{1}$ alternating with nonmagnetic $\mathrm{GGG}\left(\mathrm{Gd}_{3} \mathrm{Ga}_{5} \mathrm{O}_{12}\right)$ layers of thickness $d_{2}$. This multilayer structure, or BG MPC, is supposed to be of infinite dimensions in the $x y$ plane with the $z$ axis normal to the layers. The unit cell with the period $D$ $=d_{1}+d_{2}$ contains a YIG layer and a GGG layer. The magnetization vector in the YIG layers is oriented along the $z$ axis, as shown in Fig. 1, while we restrict ourselves to EMW propagation in the $y z$ plane.

In the multilayer structure, components of the permittivity and the permeability tensors of the YIG layers are identified with the superscript ${ }^{(1)}$, and those of the GGG layers with the superscript ${ }^{(2)}$, respectively. Because YIG is bigyrotropic, its permittivity and permeability tensors, in general, are determined by Eqs. (2) and (3) of Ref. 5. Thus, the permittivity and permeability tensors of a BG cubic YIG layer magnetized along the $z$ axis have the following nonzero components: ${ }^{14}$

$$
\begin{aligned}
& \epsilon_{x x}^{(1)}=\epsilon_{y y}^{(1)} \equiv \epsilon_{\perp}^{(1)}=\epsilon^{(1,0)}+g_{12}^{(1, e)} m_{z}^{2}, \\
& \epsilon_{z z}^{(1)} \equiv \epsilon_{\|}^{(1)}=\epsilon^{(1,0)}+g_{11}^{(1, e)} m_{z}^{2}, \\
& \epsilon_{x y}^{(1)}=\left(\epsilon_{y x}^{(1)}\right)^{*} \equiv i \epsilon^{(1)}=i f^{(1, e)} m_{z}, \\
& \mu_{x x}^{(1)}=\mu_{y y}^{(1)} \equiv \mu_{\perp}^{(1)}=\mu^{(1,0)}+g_{12}^{(1, m)} m_{z}^{2}, \\
& \mu_{z z}^{(1)} \equiv \mu_{\|}^{(1)}=\mu^{(1,0)}+g_{11}^{(1, m)} m_{z}^{2}, \\
& \mu_{x y}^{(1)}=\left(\mu_{y x}^{(1)}\right)^{*} \equiv i \mu^{\prime(1)}=i f^{(1, m)} m_{z} .
\end{aligned}
$$

Here and in the following, Gaussian units are implicit; $m_{z}$ is the $z$ component of the unit vector $\mathbf{m}=\mathbf{M} / M_{s}$, where $M_{s}$ is 


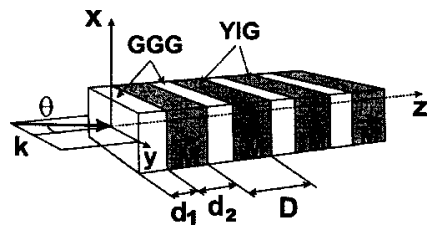

FIG. 1. Schematic of a MPC containing many periods of thickness $D$. The direction of the magnetization vector $\mathbf{M}$ in the YIG layers is shown by arrows, while the EMW propagation occurs in the $y z$ plane, with $\theta$ as the angle of incidence.

the saturation magnetization; $\epsilon^{(1,0)}$ and $\mu^{(1,0)}$, respectively, are the nonzero components of the permittivity $\epsilon_{j \ell}^{(1,0)}$ and permeability $\mu_{j \ell}^{(1,0)}$ tensors in the absence of magnetization or $\mathrm{dc}$ magnetic field; $i=\sqrt{-1}$; while $f^{(1, e(m))}, g_{11}^{(1, e(m))}$ and $g_{12}^{(1, e(m))}$ are nonzero components of the linear and the quadratic magnetooptical tensors; thus, $\epsilon^{(1,0)}=\epsilon_{x x}^{(1,0)}=\epsilon_{y y}^{(1,0)}=\epsilon_{z z}^{(1,0)}, \quad \mu^{(1,0)}$ $=\mu_{x x}^{(1,0)}=\mu_{y y}^{(1,0)}=\mu_{z z}^{(1,0)}, \quad f^{(1, e(m))}=f_{x y z}^{(1, e(m))}=-f_{y x z}^{(1, e(m))}, \quad g_{11}^{(1, e(m))}$ $=g_{z z z z}^{(1, e(m))}$, and $g_{12}^{(1, e(m))}=g_{x x z z}^{(1, e(m))}=g_{y y z z}^{(1, e(m))}$.

The permittivity and the permeability tensors of GGG are diagonal, i.e.,

$$
\epsilon_{j \ell}^{(2)}=\epsilon^{(2,0)} \delta_{j \ell}, \quad \mu_{j \ell}^{(2)}=\delta_{j \ell},
$$

where $\delta_{j \ell}$ is the Kronecker delta.

As YIG is anisotropic, EMW propagation is best described using $4 \times 4$ matrices. ${ }^{15}$ The transfer matrix (TM) method then yields the two dispersion equations ${ }^{15}$

$$
\cos \left(q_{1,2} D\right)=\frac{1}{4}\left[\operatorname{Tr}(\hat{T}) \pm \sqrt{2 \operatorname{Tr}\left(\hat{T}^{2}\right)-\operatorname{Tr}^{2}(\hat{T})+8}\right],
$$

where $q_{1}$ and $q_{2}$ are the wave numbers of the EMW in the MPC; $\hat{T}$ is the $4 \times 4 \mathrm{TM}$ of the unit cell; and $\operatorname{Tr}(\cdot)$ indicates the trace. For the two-layer unit cell under consideration, we get

$$
\hat{T}=\hat{E}_{2} \hat{S}_{12}^{-1} \hat{E}_{1} \hat{S}_{12}
$$

where the diagonal $4 \times 4$ matrix

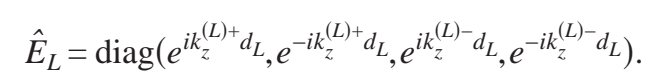

The matrix $\hat{E}_{L}$ describes a phase change inside the layer labeled $L(L=1,2),{ }^{15}$ with $k_{z}^{(L) \pm}$ as the $z$ components of the wave vectors inside that layer. The quantities $k_{z}^{(L) \pm}$ depend on the mutual orientation of the wave vector and the magnetization direction inside the particular layer, as well as on the permittivity and permeability tensor components. In the YIG layer of the unit cell,

$$
\begin{aligned}
\left(k_{z}^{(1) \pm}\right)^{2}= & -\frac{1}{2}\left[\frac{\varepsilon^{(1)}}{\varepsilon_{\|}^{(1)}}+\frac{\mu^{(1)}}{\mu_{\|}^{(1)}}\right] k_{y}^{2} \\
& -\Omega^{2}\left[\mu^{(1)} \varepsilon^{(1)}+\mu^{\prime(1)} \varepsilon^{\prime(1)}\right] \pm D^{(1)}\left[2 \varepsilon_{\|}^{(1)} \mu_{\|}^{(1)}\right]^{-1},
\end{aligned}
$$

where

$$
\begin{aligned}
\left(D^{(1)}\right)^{2}= & {\left[\mu_{\|}^{(1)} \varepsilon^{(1)}-\mu^{(1)} \varepsilon_{\|}^{(1)}\right] k_{y}^{4}-4 \mu_{\|}^{(1)} \varepsilon_{\|}^{(1)}\left[\mu^{(1)} \varepsilon^{\prime(1)}\right.} \\
& \left.+\varepsilon^{(1)} \mu^{\prime(1)}\right]\left[\mu_{\|}^{(1)} \varepsilon^{\prime(1)}+\varepsilon_{\|}^{(1)} \mu^{\prime(1)}\right] \Omega^{2} k_{y}^{2} \\
& +4 \Omega^{2}\left[\mu_{\|}^{(1)} \varepsilon_{\|}^{(1)}\right]^{2}\left[\mu^{(1)} \varepsilon^{\prime(1)}+\varepsilon^{(1)} \mu^{\prime(1)}\right]^{2},
\end{aligned}
$$
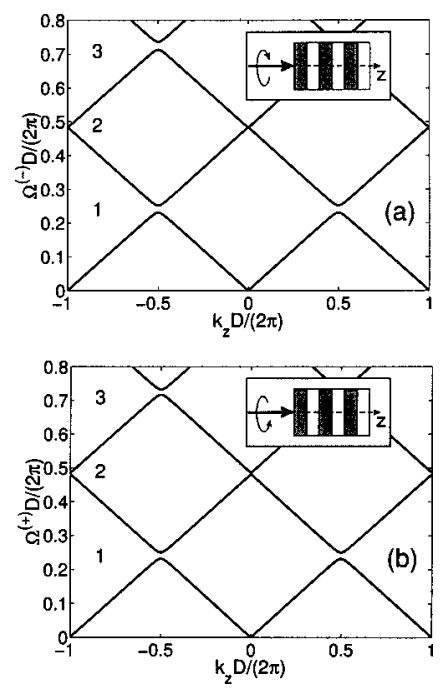

FIG. 2. Normal angular frequencies $\Omega^{( \pm)} D /(2 \pi)$ of light vs the normalized wave number $k_{z} D /(2 \pi)$ of the eigenwave inside the MPC for propagation in the $y z$ plane with tangential normalized wave number $k_{y} D /(2 \pi)=0(\theta=0)$. Upper (a) and lower (b) panels correspond to $\mathrm{LH}$ and $\mathrm{RH}$ polarized modes, as shown on the inserts. The lowest PBGs are labeled by the numbers 1, 2, and 3 .

$\Omega=\omega / c$ and $c$ is the velocity of light in vacuum. In the GGG layer of the unit cell,

$$
\left(k_{z}^{(2)}\right)^{2}=-k_{y}^{2}+\Omega^{2} \varepsilon^{(2,0)} .
$$

Matrices $\hat{S}_{12}$ in Eq. (5) connect electric and magnetic field amplitudes between adjacent YIG and GGG layers or vice versa. The components of the $4 \times 4$ matrix $\hat{S}_{12}$ are complex functions of the wave numbers $k_{z}^{(1) \pm}, k_{z}^{(2)}$, and $k_{y}$, the normalized frequency $\Omega$, as well as of the permittivity and the permeability tensors of both materials, ${ }^{15,16}$ subject to the following relationships:

$$
\begin{aligned}
& \left(\hat{S}_{12}\right)_{11}=\left(\hat{S}_{12}\right)_{22}, \quad\left(\hat{S}_{12}\right)_{12}=\left(\hat{S}_{12}\right)_{21}, \\
& \left(\hat{S}_{12}\right)_{13}=-\left(\hat{S}_{12}\right)_{24}, \quad\left(\hat{S}_{12}\right)_{14}=-\left(\hat{S}_{12}\right)_{23}, \\
& \left(\hat{S}_{12}\right)_{31}=-\left(\hat{S}_{12}\right)_{42}, \quad\left(\hat{S}_{12}\right)_{32}=-\left(\hat{S}_{12}\right)_{41}, \\
& \left(\hat{S}_{12}\right)_{34}=\left(\hat{S}_{12}\right)_{43}, \quad\left(\hat{S}_{12}\right)_{33}=\left(\hat{S}_{12}\right)_{44} .
\end{aligned}
$$

We solved Eq. (4) for a BG YIG-GGG MPC which is transparent in the NIR regime. At the wavelength $\lambda$ $=1150 \mathrm{~nm}$, YIG and GGG are characterized by the following parameters: $\quad \varepsilon^{(1,0)}=4.62, \quad f^{(1, e)} \simeq 0.02, \quad g_{11}^{(1, e)} \simeq 0.021, \quad g_{12}^{(1, e)}$ $\simeq 0.019, \mu^{(1,0)}=1.00, f^{(1, m)} \simeq 0.0077, g_{11}^{(1, m)} \simeq 0.0078, g_{12}^{(1, m)}$ $\simeq 0.0077,{ }^{13,17}$ and $\varepsilon^{(2,0)}=3.76 .{ }^{18}$ These values were used for the numerical solution of Eq. (4) for different directions of propagation in the $y z$ plane, i.e., for different values of $k_{y}$.

We computed the normalized angular frequency $\Omega^{( \pm)} D /(2 \pi)$ of light versus the normalized wave number $k_{z} D /(2 \pi)$ for different directions of light propagation inside the MPC constructed from YIG and GGG layers of equal thicknesses $\left(d_{1}=d_{2}=1 \mu \mathrm{m}\right)$. Figures 2 and 3 show the computed results for two cases: $k_{y}=0$ and $k_{y}=\pi / D$ corresponding to $\theta=0$ and $\theta \approx \pi / 6$, respectively. The three lowest PBGs are clearly evident in these figures. In Fig. 2 the PBGs at $k_{y}=0$ for the left-handed ( $\left.\mathrm{LH}\right)$ and right-handed (RH) polarized waves look similar; whereas for $k_{y}=\pi / D$ in Fig. 3, the 

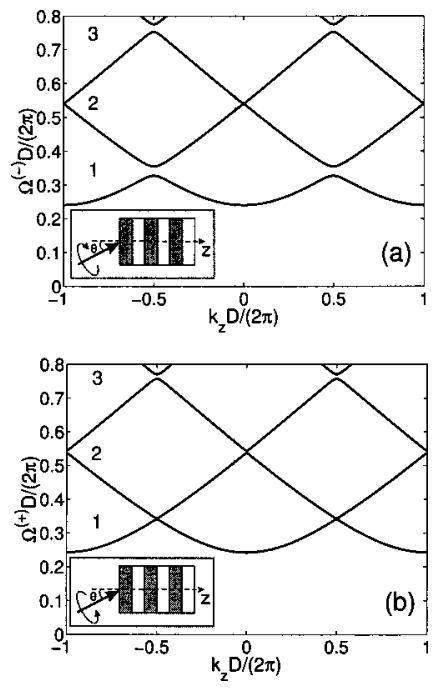

FIG. 3. The same as Fig. 2, except for $k_{y} D /(2 \pi)=0.5$ (corresponding to an angle of incidence of about $\pi / 6$ ).

PBGs with the same labels are clearly different. This means that for oblique incidence this structure displays a strong selectivity between LH and RH polarized light. For example, for LH circular polarized light, the first gap appears around $\Omega D / 2 \pi \simeq 0.33$, corresponding to a wavelength $\lambda \simeq 0.7 \mu \mathrm{m}$, whereas for $\mathrm{RH}$ circular polarization there is practically no gap. Experimentally this should be easy to verify.

The dependencies of the normalized PBG bandwidths $\Delta \Omega^{( \pm)} D / 2 \pi$ with respect to the normalized wave number $k_{y} D / 2 \pi$ are presented in Fig. 4. The absolute values of the three lowest PBG bandwidths for the LH and RH polarized EMWs are summarized in Table I. It should be mentioned that all PBGs shift upward with increasing $k_{y}$.

Clearly, Figs. 2-4 demonstrate an asymmetry between PBGs for LH and RH polarized eigenwaves in the chosen BG MPC. This asymmetry can be traced to the off-diagonal
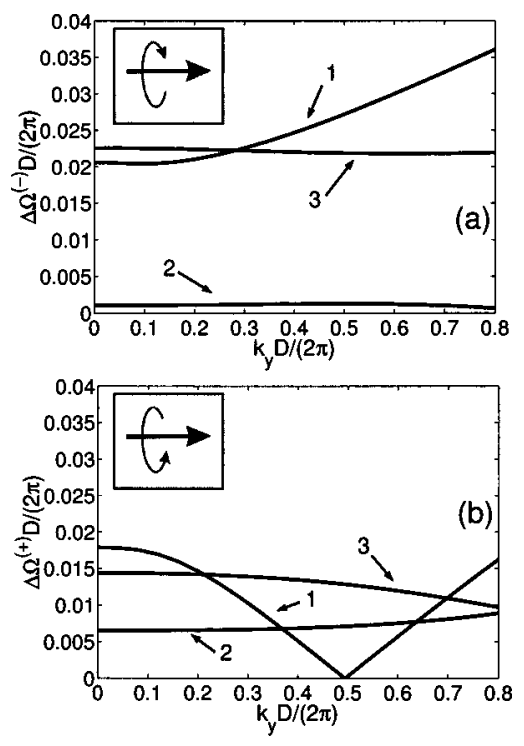

FIG. 4. Dependencies of the normalized PBG bandwidths $\Delta \Omega^{( \pm)} D /(2 \pi)$ on the direction of light propagation. Upper (a) and lower (b) panels correspond to LH and RH polarized eigenwaves shown in the inserts. The bandwidths of different PBGs are labeled as 1, 2, and 3, in accordance with PBGs labeled in Figs. 2 and 3.
TABLE I. PBG bandwidths $\Delta \Omega^{( \pm)}$for LH and RH polarized eigenwaves when $k_{y} D / 2 \pi=0 ; 0.5$. All the data for $\Delta \Omega_{n}^{( \pm)}(n=1,2,3)$ are given in $10^{14} \mathrm{~Hz}$.

\begin{tabular}{lcccccc}
\hline \hline & $\Delta \Omega_{1}^{(-)}$ & $\Delta \Omega_{1}^{(+)}$ & $\Delta \Omega_{2}^{(-)}$ & $\Delta \Omega_{2}^{(+)}$ & $\Delta \Omega_{3}^{(-)}$ & $\Delta \Omega_{3}^{(+)}$ \\
\hline$k_{y} D / 2 \pi=0$ & 1.220 & 1.058 & 0.063 & 0.386 & 1.337 & 0.852 \\
$k_{y} D / 2 \pi=0.5$ & 1.611 & 0.017 & 0.076 & 0.423 & 1.296 & 0.759 \\
\hline \hline
\end{tabular}

elements in the permittivity and the permeability tensors of YIG. Another contribution of these off-diagonal elements is the orientational dependence of the bandwidths of PBGs. On applying an external magnetic field it is possible to change the magnetization orientation in comparison with that in Fig. 1. This leads to modifications of the PBG structure, and as a result, it would be possible to tune the optical properties of MPCs with a dc magnetic field. This magnetization dependence may be used to design polarization selective tunable MO filters.

The aforementioned features of the EMW spectra can be validated by measurements of stop bands in the transmission and reflection spectra of BG MPCs, similar to calculations of transmission and reflection in BG multilayers. ${ }^{19,20}$

In conclusion, the demonstrated differences in the behavior of PBGs for LH and RH polarized EMWs may play an important role for the design of magneto-photonic devices and circuits.

This research has been done with partial support of INTAS under Grant No. 03-51-3784. I.L.L. is grateful to Radboud University of Nijmegen for the hospitality shown during his stay in Nijmegen. His visit to Nijmegen was supported by NWO and FOM.

${ }^{1}$ J. D. Joannopoulos, R. D. Meade, and J. N. Winn, Photonic Crystals: Molding the Flow of Light (Princeton University Press, Princeton, NJ, 1995).

${ }^{2}$ K. Sakoda, Optical Properties of Photonic Crystals (Springer, Berlin, 2001).

${ }^{3}$ S. G. Johnson and J. D. Joannoupolos, Photonic Crystals: The Road from Theory to Practice (Kluwer, Boston, 2002).

${ }^{4}$ A. Figotin and I. Vitebskiy, Phys. Rev. B 67, 165210 (2003).

${ }^{5}$ I. L. Lyubchanskii, N. N. Dadoenkova, M. I. Lyubchanskii, E. A. Shapovalov, and Th. Rasing, J. Phys. D 36, R277 (2003).

${ }^{6}$ M. Inoue and T. Fujii, J. Appl. Phys. 85, 5768 (1999).

${ }^{7}$ M. Levy, H. C. Yang, M. J. Steel, and J. Fujita, J. Lightwave Technol. 19, 1964 (2001).

${ }^{8}$ H. Kato, T. Matsushita, A. Takayama, M. Egava, K. Nishimura, and M. Inoue, J. Appl. Phys. 93, 3906 (2003).

${ }^{9}$ A. K. Zvezdin and V. A. Kotov, Modern Magnetooptics and Magnetooptical Materials (IOP, Bristol, 1997).

${ }^{10}$ S. E. Irvine and A. Y. Elezzabi, J. Phys. D 36, 2218 (2003).

${ }^{11}$ S. Kahl and A. M. Grishin, Appl. Phys. Lett. 84, 1438 (2004).

${ }^{12}$ G. S. Krinchik and M. V. Chetkin, Sov. Phys. JETP 9, 1368 (1959).

${ }^{13}$ A. N. Ageev, S. I. Belitskii, S. A. Kizhaev, A. S. Trifonov, and V. N. Gridnev, Sov. Phys. JETP 71, 776 (1990).

${ }^{14}$ A. G. Gurevich and G. A. Melkov, Magnetization Oscillations and Waves (CRC Press, Boca Raton, FL, 1996).

${ }^{15}$ S. B. Borisov, N. N. Dadoenkova, and I. L. Lyubchanskii, Opt. Spectrosc. 74, 670 (1993).

${ }^{16}$ I. L. Lyubchanskii, N. N. Dadoenkova, M. I. Lyubchanskii, E. A. Shapovalov, A. Lakhtakia, and Th. Rasing, in Advances in Electromagnetics of Complex Media and Metamaterials, edited by S. Zouhdi, A. Sihvola, and M. Arsalane (Kluwer, Dodrecht, 2003), p. 157.

${ }^{17}$ G. Winkler, Magnetic Garnets (Vieweg, Braunschweig, 1981).

${ }^{18}$ D. L. Wood and K. Nassan, Appl. Opt. 29, 3704 (1990).

${ }^{19}$ O. V. Ivanov and D. I. Sementsov, Pure Appl. Opt. 6, 455 (1997).

${ }^{20}$ D. G. Makarov, V. V. Danilov, and V. F. Kovalenko, Phys. Status Solidi A 201, 130 (2004). 